\title{
Atuação do profissional de educação física na saúde do trabalhador em um hospital universitário
}

\author{
Performance of the physical education professional to occupational health in a university hospital
}

\author{
Lidiane Gomes Tavares da Silva \\ Hospital Universitário de Brasilia (HUB), Universidade de \\ Brasilia (UnB), Brasilia, DF, Brasil \\ lidiane.tavares@ebserh.gov.br
}

\author{
Cariele de Aguiar Freitas \\ Universidade de Brasilia (UnB), Faculdade de Ceilândia \\ (FCE), Brasilia, DF, Brasil
}

\author{
Patrícia Azevedo Garcia \\ Universidade de Brasilia (UnB), Faculdade de Ceilândia \\ (FCE), Brasilia, DF, Brasil
}

\author{
Aline Teixeira Alves \\ Universidade de Brasilia (UnB), Faculdade de Ceilândia \\ (FCE), Brasilia, DF, Brasil
}

Liana Barbaresco Gomide Matheus

Universidade de Brasilia (UnB), Faculdade de Ceilândia

(FCE), Brasilia, DF, Brasil

\section{Resumo:}

Nas últimas décadas ocorreram diversas transformações no ambiente de trabalho que têm repercutido na saúde do trabalhador. $\mathrm{O}$ ambiente hospitalar, reconhecido como um ambiente infectocontagioso e insalubre, é também um local em que profissionais trabalham desenvolvendo atividades repetitivas, uso prolongado de um mesmo posicionamento, ritmo exaustivo de trabalho, inclusive com regime de plantões, carga horária elevada, turno noturno, sobrecarga de trabalho e outras condições de trabalho, que em médio e longo prazo podem causar diversos prejuízos funcionais. Nesse sentido, foi planejado e implementado um programa de exercícios físicos para saúde do trabalhador, com a supervisão de um profissional de educação física, direcionado aos funcionários do hospital, composto por avaliação física periódica para acompanhamento da composição corporal e exercícios Pilates e Circuito para melhoria da qualidade de vida no trabalho e realização de pesquisa científica no ambiente interdisciplinar. Esse artigo é um relato de experiência para divulgação da atuação do profissional de educação física em um hospital universitário.

PalaVras-Chave: Educação física, Hospital, Saúde do trabalhador.

\section{Abstract:}

There have been several transformations in the work environment and they have impacted the health of the worker. The hospital environment, recognized as an infectious-contagious and unhealthy environment, is also a place where there are professionals working with repetitive activities, prolonged use of the same positioning, exhaustive rhythm of work, including on-call duty, night shift, overload, productivity charge, accident risk, anti-allergic activities, and other working conditions that can cause several functional losses in the medium and long term. The Hospital implemented a Physical Exercise Program within the Worker's Health project, aimed at hospital employees, composed of periodic physical evaluation to monitor body composition and Pilates exercises and circuit to improve the quality of life and conducting scientific research in the interdisciplinary environment. This article is a report of experience to disseminate the interdisciplinary performance of the physical Education professional in a university hospital and the importance of the association of Professional Practice with science.

KEYWORDS: Physical education, Hospital, Occupational health. 


\section{INTRODUÇÃO:}

Nas últimas décadas ocorreram diversas transformações no ambiente de trabalho que têm repercutido na saúde do trabalhador. $\mathrm{O}$ desenvolvimento crescente de novas tecnologias e as inovações organizacionais modificaram a estrutura produtiva das instituições, o que acarretou mudanças significativas na forma de organização, nas condições e nas relações de trabalho (Elias \& Navarro, 2006). O desenvolvimento industrial e tecnológico levou à automação e a informatização dos postos de trabalho, sendo possíveis responsáveis pelo aumento da posição sentada nesse ambiente (Marques, Haallal, \& Gonçalves, 2010). Além disso, permanecer empregado requer ao trabalhador a capacidade de satisfazer os requisitos inerentes ao seu trabalho, de forma efetiva, o que pode ser um desafio para aqueles profissionais com lesões e/ou particularmente atuando em atividades com exigências físicas e por período prolongado. Com a tendência atual para o aumento do período de vida laboral, o menor tempo disponível para realização de exercícios físicos, a preocupação com a qualidade de vida no trabalho tem sido cada vez mais evidente(Laux, Corazza, \& Andrade, 2018; Oakman, Neupane, Proper, Kinsman, \& Nygård, 2018). Nesse sentido, estudos orientam a mudança de comportamento no sentido de evitar o sedentarismo e estimular a atividade física também no ambiente de trabalho. O sedentarismo é o quarto maior fator de risco para doenças, estando associado a 2 milhões de mortes no mundo e 5,3 milhões no Brasil, anualmente (Taiana et al., 2017). Pesquisas comprovam os benefícios à saúde dos praticantes de programas de exercício no ambiente de trabalho, como a melhoria na percepção de dores e absenteísmo, melhora de posturas(Laux et al., 2018), bem-estar psicológico e redução da obesidade(Lee et al., 2012). Dessa forma, ser ativo fisicamente no trabalho pode significar prevenção de diversas morbidades (Loitz, Potter, Walker, McLeod, \& Johnston, 2015).

A recente inclusão de Profissionais de Educação Física nos Núcleos de Apoio à Saúde da Família (NASFs) ratifica a importância do papel do Profissional de Educação Física na prevenção de doenças e promoção da saúde da população (Portaria n ${ }^{\circ} 154$, de 24 de janeiro de 2008). O ambiente hospitalar é um local em que existe profissionais que trabalham desenvolvendo atividades repetitivas, uso prolongado de um mesmo posicionamento, ritmo exaustivo de trabalho, carga horária elevada, plantões noturno e trabalhadores com mais de um emprego (Andrade, Reuter, Reckziegel, Pohl, \& Burgos, 2015). Essas e outras atividades podem acarretar morbidades musculoesqueléticas, cardiovasculares (Silva, Hotta, Silva, Almeida, \& Caromano, 2017) e metabólicas. Estudos demonstram que programas de exercícios regulares no ambiente de trabalho podem melhorar a convivência entre os seus trabalhadores e funcionam como uma forma saudável de alterar a rotina laboral, deixando-a mais dinâmica além de apresentar importantes associações entre atividade física, saúde e manutenção da capacidade funcional (Lima, Aquilas, \& Junior, 2009). Entretanto, precisa-se de maior divulgação de programas de exercícios relacionados à saúde no trabalho realizados em ambientes hospitalares.

\section{Metodologia:}

Trata-se de um relato de experiência da constituição de um Programa de Saúde do Trabalhador (PST) em um Hospital Universitário, cujo objetivo foi realizar avaliação física dos profissionais, inserir a prática de exercícios físicos no ambiente de trabalho, incentivar a continuidade dos exercícios na rotina diária do trabalhador e divulgar essa experiência para reprodutibilidade em outras instituições hospitalares. 


\section{Elaboração do Programa}

A identificação de grande número de afastamentos temporários, licenças para tratamento de doenças, absenteísmo dos profissionais de saúde e a convocação de uma profissional de educação física para a instituição culminou com a necessidade de constituição de iniciativas para melhorar a saúde do trabalhador. Iniciouse uma sondagem da profissional de educação física com os trabalhadores do hospital, o que gerou o relato de queixas ocupacionais e a sugestão de implementação e oferta de exercícios físicos no ambiente de trabalho. Foi realizado contato com professores da Faculdade de Educação Física da Universidade de Brasília (FEF - UnB), professores do Programa de Pós-graduação em Ciências da Reabilitação da Universidade de Brasília (PPGCR-UnB) e auxílio de estudantes de iniciação científica para delineamento do programa. $\mathrm{O}$ programa foi coordenado pela profissional de Educação física do hospital, em concordância com os gestores, e contou com recursos disponíveis na Unidade de Reabilitação do hospital, que foram remanejados para os horários em que não havia atendimento de pacientes. O programa consistiu na oferta de exercícios Pilates no solo, exercícios em um circuito funcional e avaliação física inicial dos funcionários do hospital. Foi considerado como critério de inclusão o desejo de participar do Programa. Foi realizada divulgação entre os diversos profissionais do hospital, nas áreas assistenciais e administrativas, e disponibilizada inscrição para os interessados em participar do programa de exercícios para saúde do trabalhador.

\section{Avaliação física}

Foi aplicado o questionário PAR-Q (Questionário de Prontidão para a Atividade física) e foram avaliados massa corporal, estatura, índice de massa corporal (IMC), circunferência de cintura e quadril para cálculo da relação cintura-quadril (RCQ), bem como percentual de gordura mensurado por meio da medida das dobras cutâneas (Rodrigues, Silva, 2006)

A massa corporal $(\mathrm{kg})$ e estatura $(\mathrm{cm})$ foram avaliados por meio de balança digital com estadiômetro (modelo P180C com indicador LD1050, LÍDER, Brasil). O índice de massa corporal (IMC) foi calculado com as medidas de peso e altura, de acordo com a fórmula IMC $=$ peso $(\mathrm{kg}) /$ altura $^{2}(\mathrm{~cm})$, sendo classificados como baixo peso (IMC < 18,5), peso normal (IMC 18,5 - 24,9), sobrepeso (IMC $\geq 25$ ), pré-obeso (IMC 25,0 - 29,9), obeso I (IMC 30,0 - 34,9), obeso II (IMC 35,0 - 39,9) e obeso III (IMC $\geq 40,0$ ) (Associação Brasileira para o Estudo da Obesidade e Síndrome Metabólica, 2009; Rezende et al., 2006). A Relação Cintura-Quadril (RCQ) foi medida com fita métrica metálica da marca Sanny, calculada com a medida do maior perímetro abdominal entre a última costela e a crista ilíaca para a circunferência de cintura e para o perímetro do quadril o maior diâmetro passando sobre os trocânteres maiores, segundo recomendações da Organização Mundial de Saúde (OMS)(Associação Brasileira para o estudo da obesidade e da síndrome metabólica (ABESO, 2016), sendo considerado risco para síndrome metabólica mulheres e homens com valores superiores a 0,85 e 0,90, respectivamente. (Associação Brasileira para o Estudo da Obesidade e Síndrome Metabólica, 2009). O percentual de gordura foi calculado a partir das medidas das dobras cutâneas, em milímetros, obtidas por meio do plicômetro (modelo clínico, CESCORF, Brasil), de forma triplicata, com precisão milimétrica, no lado direito do corpo, sendo utilizada a média das três medidas (Jackson \& Pollock, 1978; Jackson, Pollock, \& Ward, 1980).

\section{Intervenção}

Foram implementadas atividades que pudessem ser acessíveis aos trabalhadores, sendo ofertadas no intervalo do horário do almoço ou após a jornada laboral em uma sala ampla dentro do ambulatório ou área externa próxima ao estacionamento do hospital). A modalidade dos exercícios foi considerada com base 
na experiência e certificação da profissional de educação física no método, bem como o fato de demandar material de baixo custo para execução da atividade. Para o Pilates solo foram utilizados colchonete e esteira de Espuma Vinílica Acetinada (EVA), além de bola de $26 \mathrm{~cm}$ e faixa elástica. O método Pilates utilizado teve como base os princípios criados por Joseph Hubertus Pilates: respiração, controle, concentração, precisão, fluidez e centralização, e, dessa forma, a integração do corpo e mente, proporcionando consciência corporal interativa e qualificada (Mazzarino, Kerr, Wajswelner, \& Morris, 2015), estabilização do movimento dinâmico corporal e promoção de saúde física e mental. Alguns estudos demonstraram ainda melhora do índice de massa corporal (IMC) e qualidade de vida (Mazzarino et al., 2015).

Para o Circuito Funcional foram utilizados escada de agilidade, halteres emborrachados, elásticos, minibands, caixa para salto e cones pequenos. Foi implementado com base em exercícios alternados aeróbicos e anaeróbicos que envolveram diferentes grupos musculares com exercícios de resistência realizados com cargas externas e pequenos períodos de descanso (Garnacho-Castano et al., 2018). Esse tipo de exercício proporciona melhora da força, resistência, equilíbrio (Bocalini et al., 2012; Bonini-Rocha et al., 2018), bem como da composição corporal (Miller, Pearcey, Cahill, Mccarthy, et al., 2014), condicionamento cardiorrespiratório (Myers, T. R., Matthew G.S, Schneider, Schmale, M. S., 2015) e mobilidade funcional (Bonini-Rocha et al., 2018; De Luca et al., 2006).

Foi elaborado um modelo lógico para apresentação do funcionamento do Programa de exercícios para saúde do trabalhador (Figura 1) com base em propostas similares de intervenção (Cerri et al., 2016).

As aulas de Pilates solo ocorreram 4 vezes por semana (segundas e quartas-feiras, terças e quintas-feiras) com duração de até 50 minutos, no horário de intervalo de turnos (12 às 12:50 horas) ou no final da jornada de trabalho (19 às 19:50 horas) com turmas de até 20 pessoas. Já as aulas de Circuito funcional aconteceram 2 vezes por semana (segundas e quartas-feiras) com duração de até 50 minutos. A intensidade dos exercícios foi determinada por meio da Escala de Borg de 0 a 10, que controla a intensidade dos exercícios físicos a partir da percepção do próprio indivíduo, sendo utilizada durante o exercício de forma a atender os princípios de sobrecarga do treinamento. Nesse sentido, a individualidade e a percepção de esforço de cada trabalhador foi respeitada, sendo incentivados a manter uma intensidade moderada no Pilates e de moderado a intenso no Exercício Circuito(Cerri et al., 2016). 
FIGURA 1

Modelo lógico do Programa de exercícios para saúde do trabalhador.

\begin{tabular}{|c|c|c|c|c|}
\hline Recursos & Atividades & Produtos & Resultados & Objetivos \\
\hline $\begin{array}{l}\text { Materiais } \\
\text { - Instrumentos } \\
\text { de medidas } \\
\text { antropométricas } \\
\text { - Colchonete e } \\
\text { esteira de } \\
\text { Espuma } \\
\text { Vinilica } \\
\text { Acetinada } \\
\text { EVA) } \\
\text { - Bola de } 26 \mathrm{~cm} \\
\text { - Faixa elástica } \\
\text { - Escada de } \\
\text { agilidade } \\
\text { - Halteres } \\
\text { emborrachados } \\
\text { - Elásticos } \\
\text { - Mini-Bands } \\
\text { - Caixa para salto } \\
\text { - Cones } \\
\text { pequenos } \\
\text { - Caixa de som } \\
\text { - Profissional de } \\
\text { Educação Fisica } \\
\text { - Docentes da } \\
\text { UnB } \\
\text { Hecursos } \\
\text { Iniciação } \\
\text { cientifica } \\
\text { Humanos }\end{array}$ & $\begin{array}{l}\text { - Divulgação } \\
\text { nos setores } \\
\text { do hospital } \\
\text { - Divulgação } \\
\text { na intranet e } \\
\text { em murais } \\
\text { do hospital } \\
\text { - Controle de } \\
\text { presença dos } \\
\text { participantes } \\
\text { - Avaliação } \\
\text { inicial e } \\
\text { semestral } \\
\text { dos } \\
\text { participantes } \\
\text { Planejamento: } \\
\text { - Sessões de } \\
\text { exercicios } \\
\text { fisicos } \\
\text { Atividades } \\
\text { de } \\
\text { integração } \\
\text { ente os } \\
\text { participantes }\end{array}$ & $\begin{array}{l}\text { - } 109 \text { pessoas } \\
\text { avaliadas } \\
\text { - } 52 \text { pessoas } \\
\text { participaram } \\
\text { do programa } \\
\text { pelo menos } \\
\text { uma vez } \\
\text { - } 8 \text { sessões de } \\
\text { exercicios } \\
\text { fisicos } \\
\text { mensais } \\
\text { (pilates solo } \\
\text { e'ou } \\
\text { exercicios em } \\
\text { circuito) } \\
\text { - Utilização dos } \\
\text { espaços } \\
\text { disponiveis } \\
\text { dentro do } \\
\text { hospital para } \\
\text { prática da } \\
\text { atividade } \\
\text { proposta }\end{array}$ & $\begin{array}{l}\text { Curto prazo } \\
\text { - Implementação } \\
\text { do programa } \\
\text { saúde do } \\
\text { trabalhador no } \\
\text { ambiente } \\
\text { hospitalar } \\
\text { - Aumento do } \\
\text { convivio entre } \\
\text { os trabalhadores } \\
\text { participantes } \\
\text { Médio prazo } \\
\text { - Melhora do } \\
\text { conhecimento } \\
\text { acerca de } \\
\text { exercicios } \\
\text { fisicos e } \\
\text { estratégias para } \\
\text { estimulo da } \\
\text { continuação dos } \\
\text { exercicios. } \\
\text { Controle da } \\
\text { massa corporal } \\
\text { e percentual de } \\
\text { gordura } \\
\text { Longo prazo } \\
\text { - Continuidade do } \\
\text { Programa. } \\
\text { Conscientização } \\
\text { para estilo de } \\
\text { vida gaudável } \\
\text { dentro e fora do } \\
\text { ambiente de } \\
\text { trabalho }\end{array}$ & $\begin{array}{l}\text { Proporcionar } \\
\text { exercicios } \\
\text { fisicos } \\
\text { regulares no } \\
\text { ambiente de } \\
\text { trabalho para } \\
\text { melhora da } \\
\text { qualidade de } \\
\text { vida durante as } \\
\text { atividades } \\
\text { laborais, } \\
\text { incentivar a } \\
\text { prática de } \\
\text { exercicios } \\
\text { fisicos } \\
\text { regulares e a } \\
\text { promoção da } \\
\text { saude }\end{array}$ \\
\hline
\end{tabular}

Fonte: elaboração própria com base em modelo anterior (Cerri et al., 2016).

\section{Resultados E Discuss Ão}

De 1258 funcionários no hospital, 181 (14,4\%) preencheram o nome em uma lista de interessados e 109 (13,7\%) compareceram para a realização da avaliação física (Tabela 1). Apesar de nenhum trabalhador apresentar RCQ maior que o recomendado, 38\% dos avaliados apresentavam IMC maior que 25\% e 57\% dos trabalhadores apresentaram percentual de gordura maior que o esperado para a idade. 
TABELA 1

Avaliação dos trabalhadores de um ambiente hospitalar

\begin{tabular}{|c|c|c|c|c|c|c|}
\hline \multicolumn{7}{|c|}{ Mulheres } \\
\hline Faixa Etária & N & Estatura & $\begin{array}{c}\text { Massa } \\
\text { corporal }\end{array}$ & IMC & RCQ & \%Gordura \\
\hline $20-29$ anos & 33 & $162,28 \pm 5,84$ & $59,89 \pm 9,91$ & $22,75 \pm 3,53$ & $0,72 \pm 0,04$ & $26 \pm 5$ \\
$30-39$ anos & 34 & $160,93 \pm 4,57$ & $62,99 \pm 6,94$ & $24,31 \pm 2,40$ & $0,75 \pm 0,06$ & $27 \pm 5$ \\
$40-49$ anos & 22 & $159,57 \pm 5,62$ & $65,65 \pm 8,55$ & $25,79 \pm 3,19$ & $0,80 \pm 0,06$ & $28 \pm 6$ \\
$50-59$ anos & 07 & $159,86 \pm 11,55$ & $65,60 \pm 6,16$ & $25,78 \pm 2,34$ & $0,83 \pm 0,04$ & $27 \pm 3$ \\
\hline Total=96(88,07\%) \\
\hline \multicolumn{7}{|c|}{ Homens } \\
\hline Faixa Etária & N & Estatura & Massa & IMC & RCQ & $\%$ Gordura \\
\hline $20-29$ anos & 05 & $177,20 \pm 10,01$ & $86,38 \pm 21,94$ & $27,11 \pm 4,19$ & $0,85 \pm 0,07$ & $17 \pm 8$ \\
$30-39$ anos & 08 & $170,40 \pm 7,29$ & $77,39 \pm 15,55$ & $25,78 \pm 2,34$ & $0,84 \pm 0,08$ & $22 \pm 5$ \\
\hline Total=13(11,92\%)
\end{tabular}

Dos 109 trabalhadores avaliados, 52 (48\%) participaram do programa de exercícios, sendo técnicos de enfermagem, enfermeiros, médicos, fisioterapeutas, psicólogos, assistentes sociais, farmacêuticos, técnicos de saúde bucal, dentistas, terapeutas ocupacionais, assistentes e analistas administrativos. A maioria das desistências foi relacionada ao horário de trabalho contínuo, como profissionais que trabalham em escala de plantão e devido ao fato de residir longe do trabalho, o que dificultava a presença do trabalhador após a jornada laboral. A adesão maior ocorreu nas atividades oferecidas durante o intervalo de almoço, no meio da jornada de trabalho, o que não implicava a permanência por mais tempo após a jornada de trabalho.

Os trabalhadores que puderam participar das atividades de Pilates e Circuito Funcional verbalizaram melhora no condicionamento físico, bom humor, sono, bem como melhora na disposição, respiração, força muscular, coordenação motora, flexibilidade, integração social da equipe de trabalho e grande satisfação em saber que existe um programa voltado para os trabalhadores do hospital.

Um programa de exercícios pode ser uma ferramenta de prevenção de doenças e manutenção da saúde dos trabalhadores de diferentes setores (Antunes, Levandovski, Dantas, Caumo, \& Hidalgo, 2010), reduzindo a quantidade de tempo que são considerados como sedentários (Loitz et al., 2015). No Programa apresentado foi implementado o exercício Pilates que envolve a coordenação completa do corpo, mente e espírito, tendo intuito de desenvolver o corpo uniformemente, restaurar a vitalidade física, corrigir posturas erradas e revigorar a mente. Estudos demonstram que o Pilates é uma importante ferramenta para diferentes populações e disfunções e que pode melhorar o desempenho esportivo, a flexibilidade, depressão, força muscular, proporcionar reeducação postural e auxiliar na prevenção e alívio de lesões e disfunções do sistema musculoesquelético (Cruz, Liberali, Cruz, \& Netto, 2016; Mazzarino et al., 2015). Evidências científicas mostram que o Pilates tem sido utilizado para o público de trabalhadores, durante os intervalos do horário de trabalho, no sentido de contrabalancear as estruturas corporais habitualmente utilizadas nesse ambiente (E. V. Rodrigues, Gomes, Tanhoffer, \& Leite, 2014) e no intuito de reduzir o estilo de vida sedentário e melhorar o controle do estresse, melhorando a qualidade de vida (Laux et al., 2018).

O exercício em Circuito Funcional que foi utilizado nesse Programa tem apresentado benefícios em diversos estudos. Evidências demonstraram melhora na força muscular, resistência, equilíbrio corporal (Bonini-Rocha et al., 2018), bem como redução da composição corporal e melhora do condicionamento físico (Miller, Pearcey, Cahill, McCarthy, et al., 2014), sendo uma modalidade com benefícios promissores no nosso público alvo. Estudos anteriores mostraram que trabalhadores administrativos participantes do programa de exercícios de alongamento, força e treino aeróbico apresentaram diminuição dos quadros de dor lombar, com impacto na produtividade e qualidade de vida (Silva et al., 2017). Outro estudo que realizou exercícios físicos com dois grupos, sendo um no trabalho e outro em casa, relatou que a realização 
de exercícios físicos no local de trabalho foi mais eficaz na prevenção de disfunções em comparação com exercícios domiciliares (Jakobse $\mathrm{n}$ et al., 2015). Isso ressalta a importância da realização de exercícios físicos no local de trabalho.

Destaca-se que uma força de trabalho saudável e produtiva é importante para a instituição, uma vez que a capacidade de um indivíduo para executar o trabalho envolve diversos requisitos, inclusive capacidades físicas e mentais (Loitz et al., 2015), que podem ser aprimoradas com a realização de exercício físico no ambiente de trabalho e, consequentemente, contribuir para redução do número de afastamentos por doenças (Laux et al., 2018)

\section{CONSIDERAÇÕES FINAIS}

O Programa de Exercícios descrito nesse relato de experiência poderá ser utilizado como uma referência e estratégia para que outros hospitais iniciem programas semelhantes dentro do ambiente hospitalar para a prevenção e promoção da saúde do trabalhador. Ações como essa podem proporcionar um ambiente de trabalho acolhedor, com reconhecimento e empoderamento do trabalhador para o desenvolvimento de suas atividades profissionais com maior disposição, integração, socialização, e com potencial para gerar menos morbidades.

\section{ReFERENCIAS}

Andrade, A. M. De, Reuter, C. P., Reckziegel, M. B., Pohl, H. H., \& Burgos, L. T. (2015). Ginástica Laboral\#: efeitos de um programa de ginástica laboral sobre a flexibilidade em trabalhadores de diversos setores de um hospital de ensino da região do Vale do Rio Pardo / RS. Cinergis, 16(3), 209-213.

Antunes, L. C., Levandovski, R., Dantas, G., Caumo, W., \& Hidalgo, M. P. (2010). Obesity and shift work: Chronobiological aspects. Nutrition Research Reviews, 23(1), 155-168.

Associação Brasileira para o estudo da obesidade e da síndrome metabólica (ABESO). (2016). Diretrizes brasileiras de obesidade 2016. VI Diretrizes Brasileiras de Obesidade, 7-186.

Associação Brasileira para o Estudo da Obesidade e Síndrome Metabólica. (2009). Diretrizes Brasileiras da Obesidade 2009/2010. Retrieved from http://www.abeso.org.br/pdf/diretrizes_brasileiras_obesidade_2009_2010_1.pdf

Bocalini, D. S., Lima, L. S., de Andrade, S., Madureira, A., Rica, R. L., dos Santos, R. N. Pontes, F. L. (2012). Effects of circuit-based exercise programs on the body composition of elderly obese women. Clinical Interventions in Aging, 7, 551-556.

Bonini-Rocha, A. C., de Andrade, A. L. S., Moraes, A. M., Gomide Matheus, L. B., Diniz, L. R., \& Martins, W. R. (2018). Effectiveness of Circuit-Based Exercises on Gait Speed, Balance, and Functional Mobility in People Affected by Stroke: A Meta-Analysis. PM and R, 10(4), 398-409.

Cerri, N. C., Arthur, A. C., Vieira, L. M., Silva, A. E. F. da, Baba, C. T., Oliveira, I. M. Gomes, G. A. de O. (2016). Programa de Caminhada em Unidades de Saúde da Família de um contexto de alta vulnerabilidade social. Revista Brasileira de Atividade Física \& Saúde, 22(1), 100-105.

Cruz, J. C., Liberali, R., Cruz, T. M. F. da, \& Netto, M. I. A. (2016). The Pilates method in the rehabilitation of musculoskeletal disorders: a systematic review. Fisioterapia Em Movimento, 29(3), 609-622.

De Luca, V., Minganti, C., Borrione, P., Grazioli, E., Cerulli, C., Guerra, E., Kwakkel, G. (2006). Combined aerobic and resistance training in breast cancer survivors: A randomized, controlled pilot trial. International Journal of Sports Medicine, 27(7), 573-580. https://doi.org/10.1055/s-2005-865848

Elias, M. A., \& Navarro, V. L. (2006). A relação entre o trabalho, a saúde e as condições de vida: negatividade e positividade no trabalho das profissionais de enfermagem de um hospital escola. Revista Latino-Americana de Enfermagem, 14(4), 517-525. 
Garnacho-Castano, M. V., Domínguez, R., Muñoz González, A., Feliu-Ruano, R., Serra-Payá, N., \& Maté-Muñoz, J. L. (2018). Exercise Prescription Using the Borg Rating of Perceived Exertion to Improve Fitness. International Journal of Sports Medicine, 39(2), 115-123.

Jackson, A. S. \& Pollock, M. L. (1978). Generalized equations for predicting body density of men. British journal of nutrition, 40(3), 497-504.

Jackson, A. S., Pollock, M. L. \& Ward, A. N. N. (1980). Generalized equations for predicting body density of women. Medicine and science in sports and exercise, 12(3), 175-181.

Jakobsen, M. D., Sundstrup, E., Brandt, M., Jay, K., Aagaard, P., \& Andersen, L. L. (2015). Physical exercise at the workplace prevents deterioration of work ability among healthcare workers: Cluster randomized controlled trial. BMC Public Health, 15(1), 1-9.

Laux, R. C., Corazza, S. T., \& Andrade, A. (2018). Workplace physical activity program: An intervention proposal. Revista Brasileira de Medicina Do Esporte, 24(3), 238-242.

Lee, I. M., Shiroma, E. J., Lobelo, F., Puska, P., Blair, S. N., Katzmarzyk, P. T., Wells, J. C. (2012). Effect of physical inactivity on major non-communicable diseases worldwide: An analysis of burden of disease and life expectancy. The Lancet, 380(9838), 219-229.

Lima, V. A. de, Aquilas, A. L., \& Junior, M. F. (2009). Efeitos de um programa de exercícios físicos no local de trabalho sobre a flexibilidade e percepção de dor musculoesquelética entre trabalhadores de escritório. Revista Brasileria de Medicina do Trabalho, 7, 11-17.

Loitz, C. C., Potter, R. J., Walker, J. L., McLeod, N. C., \& Johnston, N. J. (2015). The effectiveness of workplace interventions to increase physical activity and decrease sedentary behaviour in adults: Protocol for a systematic review. Systematic Reviews, 4(1), 1-8.

Marques, N. R., Haallal, C. Z., \& Gonçalves, M. (2010). Características biomecânicas, ergonômicas e clínicas da postura sentada: uma revisão. Fisioterapia e Pesquisa, 17(3), 270-276.

Mazzarino, M., Kerr, D., Wajswelner, H., \& Morris, M. E. (2015). Pilates Method for Women's Health: Systematic Review of Randomized Controlled Trials. Archives of Physical Medicine and Rehabilitation, 96(12), 2231-2242.

Miller, M. B., Pearcey, G. E. P., Cahill, F., McCarthy, H., Stratton, S. B. D., Noftall, J. C., Button, D. C. (2014). The Effect of a Short-Term High-Intensity Circuit Training Program on Work Capacity, Body Composition, and Blood Profiles in Sedentary Obese Men: A Pilot Study. BioMed Research International, 2014, 1-10.

Myers, T. R., Schneider, M. G., Schmale, M. S., \& Hazell, T. J. (2015). Whole-body aerobic resistance training circuit improves aerobic fitness and muscle strength in sedentary young females. The Journal of Strength \& Conditioning Research, 29(6), 1592-1600.

Oakman, J., Neupane, S., Proper, K. I., Kinsman, N., \& Nygård, C. H. (2018). Workplace interventions to improve work ability: A systematic review and meta-analysis of their effectiveness. Scandinavian Journal of Work, Environment and Health, 44(2), 134-146.

Rezende, F. A. C., Rosado, L. E. F. P. L., Ribeiro, R. D. C. L., Vidigal, F. D. C., Vasques, A. C. J., Bonard, I. S., \& Carvalho, C. R. De. (2006). Índice De Massa Corporal E Circunferência Abdominal: Associação Com Fatores De Risco Cardiovascular. Arquivos Brasileiros de Cardiologia, 87(6), 728-734.

Rodrigues, E. V., Gomes, A. R. S., Tanhoffer, A. I. P., \& Leite, N. (2014). Effects of exercise on pain of musculoskeletal disorders: a systematic review. Acta Ortopédica Brasileira, 22(6), 334-338.

Rodrigues, M. N., Silva, S. C. da, Monteiro, W. D., \& Farinatti, P. de T. V. (2006). Estimativa da gordura corporal através de equipamentos de bioimpedância, dobras cutâneas e pesagem hidrostática. Revista Brasileira de Medicina Do Esporte, 7(4), 125-131.

Silva, J. A. M. G., Hotta, T. T. H., Silva, T. H. da, Almeida, M. H. M. de, \& Caromano, F. A. (2017). Desenvolvimento de um programa de promoção da saúde para trabalhadores administrativos. Revista Saúde e Pesquisa, 10(3), 557566.

Taiana, P., Sousa, D. M., Roberta, A., Sousa, R. De, Sene, E., Tainara, G., \& Sousa, D. M. (2017). Sedentary behavior among professionals in the family health strategy. Revista de Enfermagem da UFPI, 6(3), 24-29. 